\title{
Is botulinum toxin type A more effective and safer than other treatments for the management of lower limb spasticity in children with cerebral palsy? A Cochrane Review summary with commentary
}

\author{
Heakyung $\mathrm{Kim}^{\mathrm{a}, *}$ and Kat Kolaski ${ }^{\mathrm{b}, *}$ \\ ${ }^{a}$ Department of Rehabilitation and Regenerative Medicine, Columbia University Irving Medical Center, \\ New York, NY, USA \\ ${ }^{\mathrm{b}}$ Departments of Orthopedics and Pediatrics, Wake Forest Baptist Health, Comp Rehab, \\ Winston-Salem, NC, USA
}

\begin{abstract}
.
BACKGROUND: Botulinum toxin A (BoNT-A) is a well-accepted treatment for the medical management of spasticity in children with cerebral palsy $(\mathrm{CP})$.

OBJECTIVE: To assess the efficacy and safety of BoNT-A compared with other treatment options in managing lower limb spasticity in children with $\mathrm{CP}$.

METHODS: A summary of the Cochrane Review update by Blumetti et al. (2019), with comments.

RESULTS: This review included 31 randomized controlled trials (1508 participants). Compared with usual care/physiotherapy, the evidence is very uncertain about the effect of BoNT-A on gait, function, ankle joint range of motion (ROM), satisfaction, and ankle spasticity in children with CP. Compared with placebo/sham, BoNT-A probably benefits these same outcomes, although the results for function are contradictory. BoNT-A may not be more effective than serial casting at improving gait, function, ankle ROM and spasticity at any time point. However, it may be more effective than an orthosis at medium-term follow-up for hip ROM and adductor spasticity, but not function. The rate of adverse events with BoNT-A is similar to placebo/sham and serial casting.

CONCLUSIONS: Evidence for the effectiveness and safety of BoNT-A for the management of lower limb spasticity in children with $\mathrm{CP}$ is uncertain, with better quality evidence available from studies of placebo/sham than non-placebo controls. To produce high-quality evidence, future studies need to improve their methodological quality and increase sample sizes.
\end{abstract}

Keywords: Botulinum toxin A, spasticity, efficacy, safety, evidence

\footnotetext{
*Address for correspondence: Heakyung Kim, Department of Rehabilitation \& Regenerative Medicine, Columbia University Irving Medical Center, 180 Fort Washington Ave, HP-165, New York, NY 10032, USA. Tel.: +1 212305 5337; E-mail:
}

hk2641@cumc.columbia.edu. and Kat Kolaski, Departments of Orthopedics and Pediatrics, Wake Forest Baptist Health, Comp Rehab, 131 Miller St, Winston-Salem, NC 27103, USA. Tel.: +1 704716 4348; E-mail: kkolaski@wakehealth.edu. 
The aim of this commentary is to discuss, from a rehabilitation perspective, the published Cochrane Review "Botulinum toxin type A in the treatment lower limb spasticity in children with cerebral palsy"1 by Blumetti, Belloti, Tamaoki, \& Pinto (2019) ${ }^{\mathrm{a}}$, under the direct supervision of the Cochrane Developmental, Psychosocial and Learning Problems Review Group. This Cochrane Corner is produced in agreement with NeuroRehabilitation by Cochrane Rehabilitation.

a This summary is based on a Cochrane Review previously published in the Cochrane Database of Systematic Reviews 2019, Issue 10. Art No.: CD001408. DOI: 10.1002/14651858.CD001408. pub2 (see www.cochranelibrary.com for information). Cochrane Reviews are regularly updated as new evidence emerges and in response to feedback, and Cochrane Database of Systematic Reviews should be consulted for the most recent version of the review. The views expressed in the summary with commentary are those of the Cochrane Corner author and do not represent the Cochrane Library or Wiley.

\section{Background}

Botulinum toxin type A (BoNT-A) is the first-line medical treatment for focal spasticity in children with cerebral palsy (CP). BoNT-A injected into the muscle decreases spasticity by causing a local paralysis due to blockade of neuromuscular transmission. A Cochrane Review assessed the efficacy and safety of BoNT-A for the treatment of lower limb spasticity in children with CP (Blumetti et al., 2019). It updated the original review published in 2000 (Ade-Hall \& Moore, 2000).

Botulinum toxin type $\mathrm{A}$ in the treatment of lower limb spasticity in children with cerebral palsy (Blumetti, Belloti, Tamaoki, \& Pinto, 2019)

\section{Objective}

The aim of this Cochrane Systematic Review was to compare the efficacy and safety of BoNT-A with other treatments in the management of lower limb spasticity in children with $\mathrm{CP}$.

\section{What was studied and methods}

Children with CP, aged between birth and 19 years, who had been treated for lower limb spas- ticity. The intervention was BoNT-A injections into the lower limb muscles. Comparators were usual care/physiotherapy, placebo/sham, serial casting and orthoses. The primary outcomes were gait (instrumented and observational) analysis and function; secondary outcomes were joint range of motion (ROM), quality of life, caregiver satisfaction, spasticity, and adverse events (AE). Outcomes were analyzed at short-term ( 2 to 8 weeks), medium-term (12 to 16 weeks), and long-term ( $>24$ weeks) follow-up.

\section{Results}

This review included 31 randomized controlled trials (RCTs; 28 new to this update), with 1508 participants (mostly males; mean ages between 3 and 7 years). Below, we summarize the results of the clinically important outcomes reported in the 'Summary of findings' tables by Blumetti et al. for which there were data. The evidence is current to October 2018.

Comparison 1: BoNT-A versus usual
care/physiotherapy
BoNT-A may improve:

- observational gait scores at medium-term follow-up (1 RCT, 40 participants);

- function at short-term (2 RCTs, 123 participants), medium-term (4 RCTs, 191 participants), and long-term (3 RCTs, 119 participants) follow-up;

- ankle ROM at short-term (2 RCTs, 186 assessments), medium-term (5 RCTs, 272 assessments) and long-term (4 RCTs, 250 assessments) follow-up;

- satisfaction at long-term follow-up (1 RCT, 24 participants); and

- ankle spasticity at short-term (2 RCTs, 186 assessments), medium-term (3 RCTs, 226 assessments), and long-term (4 RCTs, 258 assessments) follow-up (all very low-quality evidence).

The proportion of AE with BoNT-A at short-term follow-up was 37\%; (5 RCTs, 206 participants; very low-quality evidence).

\section{Comparison 2: BoNT-A versus placebo/sham BoNT-A probably improves:}

- instrumented gait scores at short-term follow-up (1 RCT, 19 participants); 
- observational gait scores at short-term (4 RCTs, 261 assessments) and medium-term (3 RCTs, 248 assessments) follow-up;

- function at medium-term (5 RCTs, 327 participants), but not short-term (4 RCTs, 305 participants) or long-term (2 RCTs, 91 participants) follow-up;

- ankle ROM at short-term (3 RCTs, 291 assessments), medium-term (2 RCTs, 150 assessments) and long-term (1 RCT, 40 assessments) follow-up;

- satisfaction at short-term (1 RCT, 41 assessments), medium-term (2 RCTs, 74 assessments) and long-term (1 RCT, 33 assessments) followup; and

- ankle spasticity (1 RCT) at short-term (156 assessments), medium-term (144 assessments) and long-term (42 assessments) follow-up (all moderate-quality evidence).

The rate of AEs at short-term follow-up did not differ between groups (12 RCTs, 918 participants; moderate-quality evidence).

Comparison 3: BoNT-A versus serial casting

BoNT-A may not be more effective at improving:

- instrumented gait scores at short-term (1 RCT, 21 participants) and medium-term follow-up (3 RCTs, 61 participants);

- ankle ROM at short-term (1 RCT, 18 assessments) and medium-term (3 RCTs, 67 assessments) follow-up; and

- ankle spasticity at short-term (1 RCT, 18 assessments) and medium-term (3 RCTs, 67 assessments) follow-up (all low-quality evidence).

BoNT-A is probably not more effective at improving:

- instrumented gait scores at long-term follow-up (1 RCT, 26 participants);

- observational gait scores at short-term, mediumterm and long-term follow-up (1 RCT, 18 participants);

- function at short-term (1 RCT, 18 assessments), medium-term (2 RCTs, 41 assessments); and long-term (1 RCT, 18 assessments) follow-up; and

- ankle ROM and ankle spasticity (2 RCTs, 44 assessments) at long-term follow-up (all moderate-quality evidence).
BoNT-A may not lead to a higher rate of AEs at short-term follow-up (3 studies, 46 participants; lowquality evidence).

Comparison 4: BoNT-A versus orthoses with Johnstone pressure splints

At medium-term follow-up, there was no evidence of a difference in function between the groups, though BoNT-A may be more effective for improving hip ROM and hip spasticity (1 RCT, 43 participants; all very low-quality evidence).

\section{Conclusions}

There is limited evidence that BoNT-A is more effective than a non-placebo control at improving gait, function, ROM, satisfaction and spasticity, but moderate-quality evidence that it is probably more effective than placebo/sham. The rate of adverse events with BoNT-A was similar to placebo/sham and serial casting. Imprecision (due to the limited number of studies and/ participants), risk of bias (most commonly from inadequate blinding and allocation concealment procedures), and heterogeneity of the RTCs downgraded the quality of this body of evidence.

\subsection{Implications for practice in neurorehabilitation}

This review compared the efficacy of BoNT-A to placebo and three different treatment options for the management of lower limb spasticity in children with $\mathrm{CP}$, and found no high-quality evidence in favour of BoNT-A. There was moderate-quality evidence that BoNT-A is probably more effective than placebo/sham for gait, function, ROM, satisfaction and spasticity. This is to be expected. Clinicians may be especially interested in the evidence that shows limited effectiveness of BoNT-A compared to usual care/physiotherapy, as well as evidence showing BoNT-A is probably not more effective than serial casting for gait, function, ROM, and spasticity. These findings may encourage clinicians to maximize nonmedical treatments before considering BoNT-A. In countries where BoNT-A is not readily available or where its use is limited by costs, application of these other treatment options may be equally effective.

The indications for certain spasticity treatments, including BoNT-A, may change over time, especially in a developing child. In current practice, clinicians 
who manage spasticity in children with $\mathrm{CP}$ are likely to use a combination of different treatments, rather than relying one. Ideally, this multimodal treatment approach will be acknowledged in future studies of BoNT-A, which should also assess outcomes using valid and reliable measures that reflect the content of the International Classification of Functioning, Disability and Health (ICF) Core Sets for children and youth with CP (Schiariti et al. 2017).

\section{Acknowledgments}

The authors thank Cochrane Rehabilitation and Cochrane Developmental, Psychosocial and Learning Problems for reviewing the contents of the Cochrane Corner.

\section{Conflict of interest}

HK has received research grants from Allergan and Ipsen, was an Allergan and Ipsen Pediatric Spasticity advisory member and is a consultant for Allergan and
Merz. KK is a speaker for Piramal. This research did not receive any specific grant from funding agencies in the public, commercial, or not-for-profit sectors.

\section{References}

Ade-Hall, R. A., \& Moore, A. P. (2000). Botulinum toxin A in the treatment of lower limb spasticity in cerebral palsy. Cochrane database Systematic Reviews, (2), CD001408. CD001408. DOI: 10.1002/14651858. PMID: 10796784

Blumetti, F. C., Belloti, J. C., Tamaoki, M. J., \& Pinto, J. A. (2019). Botulinum toxin type A in the treatment of lower limb spasticity in children with cerebral palsy. Cochrane Database of Systematic Reviews, (10), CD001408. DOI: 10.1002/14651858. CD001408.pub2

Schiariti, V., Tatla, S., Sauve, K., \& O’Donnel,1 M. (2017). Toolbox of multiple-item measures aligning with the ICF Core Sets for children and youth with cerebral palsy. European Journal of Paediatric Neurology, 21(2), 252-263. DOI: 10.1016/j.ejpn.2016.10.007. PMID: 27864012 